\title{
Eficacia del mini mental y PFEIFFER (SPMSQ) para detectar deterioro cognitivo en mayores de 65 años
}

\author{
Eficácia do mini mental e PFEIFFER (SPMSQ) para detectar deterioração cognitiva em mais de \\ 65 anos
}

\section{Detecção de mutações no gene 235 do Helicobacter pylori envolvidas na resistência à claritromicina}

\author{
Daniela Elizabeth Angamarca Coello ${ }^{1}$ \\ dani_ang19@hotmail.com \\ ORCID: 0000-0003-4930-5086
}
Danilo Gustavo Muñoz Palomeque ${ }^{3}$
dmunozp@ucacue.edu.ec
ORCID: 0000-0002-9800-8363

\author{
Álvaro González Ortega ${ }^{2}$ \\ agonzalezo@ucacue.edu.ec \\ ORCID: 0000-0001-7483-6845
}
Juan Diego Domínguez Villizhañay ${ }^{1}$
jdominguezv@ucacue.edu.ec ORCID: 0000-0002-7880-4354

1 Ministerio de Salud Pública del Ecuador. Centro de Salud de Ricaurte. Ecuador

2Ministerio Salud Publica del Ecuador. Docente de la Universidad Católica de Cuenca, Ecuador

${ }^{3}$ Docente de la Universidad Católica de Cuenca. Departamento de Investigación de la Universidad Católica de Cuenca, Ecuador

Recibido 10 de septiembre 2020 | Arbitrado y aceptado 01 de octubre 2020 | Publicado 22 de diciembre 2020

\begin{abstract}
RESUMEN
Introducción: El deterioro cognitivo (DC) es un problema cada vez más frecuente, y a menudo no es diagnosticado, porque impide una terapéutica temprana, dificultando posteriormente la calidad de vida de quien lo padece. Objetivo: determinar la eficacia de los cuestionarios Mini Mental y PFEIFFER (SPMSQ) para detectar la existencia de deterioro cognitivo en personas mayores de 65 años. Método: estudio analítico de corte transversal. La muestra estuvo constituida por los adultos mayores del centro geriátrico de Macas. La utilidad diagnóstica se evaluó mediante el cálculo de área bajo la curva ROC (AUC), para el cálculo de los valores de sensibilidad (S), especificidad (E) se tomaron los puntos de corte más significativos y se comparó ambos test para determinar DC. Resultados: el $53 \%$ de los pacientes fueron del género masculino y la media de 82 años. El $50 \%$ presentaron síntomas de DC con al menos un cuestionario positivo; el $58 \%$ fueron positivos con Pfeiffer y el $91 \%$ con Mini mental. Finalmente, los valores de sensibilidad y especificidad del mini mental fueron de $91 \%$ y $100 \%$ respectivamente y el AUC fue de 1 mientras Pfeiffer obtuvo una $\mathrm{S}$ y E de $100 \%$ y AUC de 0.9, demostrando este último ser más efectivo para el diagnóstico de DC. Conclusión: se determinó que el test de Pfeiffer fue más efectivo que el Mini mental para el diagnóstico de DC; además, la edad y el nivel de escolaridad son factores más asociados a esta patología.
\end{abstract}

Palabras clave: Eficacia; mini mental; Pfeiffer; deterioro cognitivo; adulto mayor

\begin{abstract}
Introduction: Cognitive impairment (CD) is an increasingly frequent problem, and it is often not diagnosed, because it prevents early therapy, later hindering the quality of life of those who suffer from it. Objective: to determine the effectiveness of the Mini Mental and PFEIFFER questionnaires (SPMSQ) to detect the existence of cognitive impairment in people over 65 years of age. Method: analytical cross-sectional study. The sample consisted of the elderly from the Macas geriatric center. The diagnostic utility was evaluated by calculating the area under the ROC curve (AUC), for the calculation of the sensitivity $(S)$, specificity $(E)$ values, the most significant cut-off points were taken and both tests were compared to determine DC. Results: $53 \%$ of the patients were male and the average was 82 years old. $50 \%$ presented symptoms of CD with at least one positive questionnaire; $58 \%$ were positive with Pfeiffer and $91 \%$ with Mini mental. Finally, the sensitivity and specificity values of the mini mental were $91 \%$ and $100 \%$ respectively and the AUC was 1 while Pfeiffer obtained an S and $E$ of $100 \%$ and AUC of 0.9 , proving the latter to be more effective for the diagnosis of DC. Conclusion: it was determined that the Pfeiffer test was more effective than the Mini mental for the diagnosis of $C D$; Furthermore, age and level of education are factors most associated with this pathology.
\end{abstract}

DA: Medica General Graduada De La Universidad Católica De Cuenca Universidad Católica de Cuenca. Ecuador.

AG: Doctor en Medicina y Cirugía, Diplomado Superior En Desarrollo Local y Salud, Especialista en Medicina Interna Especialista en Cerencia y Planificación Estratégica en Salud, trat Estratégica en Salud, tratante del Hospital Vicente Corral Moscoso y Catedrático de la Facultad de Medicina de la Universidad Católica de Cuenca. Universidad Católica de Cuenca. Ecuador.

DM: Doctor en Medicina y Cirugía Especialista en Medicina Interna, Catedrático de la Facultad de Medicina de la Universidad Católica de Cuenca. Universidad Católica de Cuenca. Ecuador.

ID: Médico Cirujano por Universidad JD: Médico Cirujano por Universidad
Tecnológica Equinoccial (2014), Médico Tecnológica Equinoccial (2014), Médico
residente del servicio de Cirugía General del residente del servicio de Cirugía General del Hospital General Riobamba IESS desde el año 2016. Instituto Ecuatoriano de Riobamba. Ecuado

Key words: Efficacy; mini mental; Pfeiffer; cognitive impairment; older adult 
DA: Medica General Graduada De La Universidad Católica De Cuenca. Universidad Católica de Cuenca. Ecuador.

AG: Doctor en Medicina y Cirugía Diplomado Superior En Desarrollo Local y Salud, Especialista en Medicina Interna, Salud, Especialista en Medicina Interna,
Especialista en Gerencia y Planificación Especialista en Gerencia y Planificación Vicente Corral Moscoso y Catedrático de la Facultad de Medicina de la Universidad Católica de Cuenca. Universidad Católica de Cuenca. Ecuador.

DM: Doctor en Medicina y Cirugía Especialista en Medicina Interna, Catedrático de la Facultad de Medicina de la Universidad Católica de Cuenca Universidad Católica de Cuenca. Ecuador.

JD: Médico Cirujano por Universidad Tecnológica Equinoccial (2014), Médico residente del servicio de Cirugía General del Hospital General Riobamba IESS desde el año 2016. Instituto Ecuatoriano de Seguridad Social - Hospital General Riobamba. Ecuador

\section{RESUMO}

Introdução: O déficit cognitivo (DC) é um problema cada vez mais frequente, muitas vezes não diagnosticado, pois impede a terapia precoce, prejudicando posteriormente a qualidade de vida de quem a sofre. Objetivo: determinar a eficácia dos questionários Mini Mental e PFEIFFER (SPMSQ) para detectar a existência de déficit cognitivo em pessoas com mais de 65 anos. Método: estudo transversal analítico. A amostra foi composta por idosos do centro geriátrico de Macas. A utilidade diagnóstica foi avaliada pelo cálculo da área sob a curva ROC (AUC), para o cálculo dos valores de sensibilidade (S), especificidade $(E)$, os pontos de corte mais significativos foram tomados e ambos os testes foram comparados para determinar DC. Resultados: $53 \%$ dos pacientes eram do sexo masculino e a média de idade foi de 82 anos. $50 \%$ apresentaram sintomas de DC com pelo menos um questionário positivo; $58 \%$ foram positivos com Pfeiffer e $91 \%$ com Mini mental. Finalmente, os valores de sensibilidade e especificidade do mini mental foram $91 \%$ e $100 \%$ respectivamente e a AUC foi 1 enquanto Pfeiffer obteve S e E de 100\% e AUC de 0,9, provando que esta última é mais eficaz para o diagnóstico de DC. Conclusão: determinouse que o teste de Pfeiffer foi mais eficaz do que o Mini mental para o diagnóstico de DC; além disso, a idade e o nível de escolaridade são os fatores mais associados a essa patologia.

Palavras-chave: Eficácia; mini mental; Pfeiffer; comprometimento cognitivo; idoso

\section{INTRODUCCIÓN}

$\mathrm{E}$ I deterioro cognitivo (DC) inicia en el envejecimiento, definido como la pérdida de funciones cognitivas caracterizada por disminución de la memoria, juicio y de la atención. Puede ocurrir debido a problemas neurodegenerativos, vasculares $\mathrm{y}$ de distimia o disforia (1), depende de varios factores tanto fisiológicos (enfermedades crónicas degenerativas) como ambientales (ejercicio físico, estado anímico) y está sujeto a una gran variabilidad interindividual (2).

Según la INEC en el Ecuador existen 1.229.089 adultos mayores de los cuales $53,4 \%$ son mujeres, además se observa un crecimiento del $2,5 \%$, se prevé que para el 2020 el porcentaje de adultos mayores será de 7,4 \% y para el 2054 representará el 18\% de la población (1).

La pérdida de la capacidad personal de realizar actividades puede ser la única manifestación de este proceso (3). La evaluación del deterioro cognitivo debe fundamentarse principalmente en una historia clínica detallada valorando los cambios cognitivos y funcionales, la evaluación neuropsicológica, funcional y neuropsiquiátrica $\mathrm{y}$, por último, la exploración neurológica y las pruebas complementarias recomendadas. Se encontró que los síntomas psicológicos y conductuales que tienen mayor asociación con el DCL son depresión, irritabilidad, ansiedad, apatía y agitación (4)

Por lo descrito, se ha visto la necesidad de la utilización de test breves y sencillos. Los instrumentos que se utilicen con este fin deben reunir unas condiciones de aplicabilidad (brevedad, simplicidad, aceptabilidad, etc.) y unos mínimos requisitos psicométricos (validez, fiabilidad, etc.)

En este estudio se determinar la eficacia diagnóstica entre los cuestionarios Mini Mental y PFEIFFER (SPMSQ), con lo cual se espera poder contribuir así a un diagnóstico más eficiente y preciso, para una actuación terapéutica temprana que evite o retrase la aparición de la demencia y reduzca su elevado coste personal, familiar y social. 


\section{MATERIALES Y MÉTODOS}

$\mathrm{L}$ a investigación se efectuó con un diseño no experimental de tipo analítico de corte transversal, en el centro geriátrico nuestra señora Purísima de Macas, escogido por su alta población asignada. Se seleccionó a los adultos mayores que cumplieron con los siguientes criterios de inclusión: tener más de 65 años, cualquier género, firma del consentimiento informado, usuarios del centro geriátrico Nuestra Señora Purísima de Macas. Posteriormente, se entregaron de manera física ambos cuestionarios y se explicó el proceso de llenado. El cuestionario del Estado Mental Breve de Pfeiffer (SPMSQ): instrumento de aplicación rápida que no requiere preparación especial, consta de 10 preguntas y es útil en personas de edad avanzada y analfabetas. Se contabilizan los errores, como punto de corte para deterioro cognitivo 3 o más en personas escolarizadas y 4 o más para analfabetas; mientras el cuestionario mini mental consta en su estructura de 30 puntos totales el punto de corte establecido se fijó en 24 , no es aplicable analfabetos; lo que obliga a realizar correcciones; además se obtuvieron características sociodemográficas de los pacientes como edad, sexo, nivel de escolaridad.

\section{RESULTADOS}

$\mathrm{S}$ e aplicaron el mini mental y el SPMSQ a 55 adultos mayores, con un porcentaje similar del género femenino como masculino, 26 (47\%) y $29(57 \%)$ respectivamente, con una media de 82 años, el grupo etario mayoritario fue el de 75 - 90 años con 30 (55\%), en cuanto al nivel de estudios encontramos que primaria representa aproximadamente el $65 \%$ de la población. Los datos de las variables socioeconómicas se aprecian en la tabla 1.

Tabla 1. Características sociodemográficas de los 55 adultos mayores del centro geriátrico Nuestra Señora Purísima del cantón Macas.

\begin{tabular}{lccc}
\hline & Características & Adultos mayores n 55 & $\mathbf{\% ~ 1 0 0}$ \\
\hline \multirow{3}{*}{ Edad } & $65-74$ & 12 & 22 \\
& $75-90$ & 30 & 55 \\
\multirow{3}{*}{ Sexo } & $>90$ & 13 & 24 \\
& Femenino & 26 & 47 \\
\multirow{3}{*}{ Nivel de estudio } & Masculino & 29 & 53 \\
& Ninguno & 9 & 16 \\
& Primaria & 36 & 65 \\
& Secundaria & 10 & 18 \\
\hline
\end{tabular}




\section{Cuestionario mini mental}

Del total de la muestra estudiada (55), el $91 \%$ de los adultos mayores institucionalizados tienen deterioro cognitivo, de los cuales 26(52\%) son del sexo masculino, 24(48\%) pertenecen al sexo femenino, además presentan con mayor frecuencia un nivel de escolaridad primaria representado el 32 (64\%). Tabla 2

Se estima que los adultos mayores de entre 65 a 76 años presentan en promedio una puntuación de 22 , entre 77 a 86 años una puntuación de 18, entre 87 a 96 años 14 puntos, por último, entre 97 a 106 años 5 puntos.

\section{Cuestionario Pfeiffer}

Se encontró que 32 (58\%) adultos mayores presentaron puntuación menor a 6 , siendo compatible con deterioro cognitivo y los 23 (42\%) individuos restantes puntuación mayor o igual a 6, resultando negativo.

Del 58\% que resultó positivo para DC se evidencio al sexo femenino con el 17 (53\%), mientras el 15 (47\%) pertenecen al sexo masculino, se encontró que tuvo acceso a nivel de educación primaria representado el 21 (66\%). Tabla 2.

Con respecto a la edad, de los casos positivos, agrupados en rangos entre 65 a 76 años presentaron una puntuación de 7 , y con un promedio similar los adultos mayores que se encuentran entre 77 - 96 años, además se encuentra un promedio de 0 entre las edades de 97 a 106 años.

Tabla 2. Características sociodemográficas y resultados por grupos diagnósticos.

\begin{tabular}{llcccc}
\hline & & $\begin{array}{c}\text { DC Mini } \\
\text { mental }\end{array}$ & $\begin{array}{c}\text { NDC } \\
\text { Mini mental }\end{array}$ & DC SPMSQ & NDC SPMSQ) \\
\hline \multirow{3}{*}{ Sexo } & Masculino & 26 & 3 & 15 & 14 \\
& Femenino & 24 & 2 & 17 & 9 \\
& Total & 50 & 5 & 32 & 23 \\
& Analfabetos & 9 & 0 & 7 & 2 \\
Nivel de & Primaria & 32 & 4 & 21 & 15 \\
escolaridad & Secundaria & 9 & 1 & 4 & 6 \\
& Total & 50 & 5 & 32 & 23 \\
\multirow{2}{*}{ DC: deterioro } & cognitivo NDC: no deterioro cognitivo & & & \\
\hline
\end{tabular}

\section{Curvas ROC}

La eficacia se evaluó mediante el cálculo del área bajo la curva ROC, así como con el cálculo de los valores de sensibilidad, especificidad para los puntos de corte más significativos. Se ha analizado de forma independiente el cuestionario Pfeiffer (SPMSQ) como el cuestionario mini mental.
La tabla 3, muestra los valores de Sensibilidad y Especificidad, para distintos puntos de corte del cuestionario mini mental para el diagnóstico de DC; el punto de corte que maximiza S y E es 30/24 (24 positivo/25 negativo), para el cual se obtiene una $\mathrm{S}$ de 0,91 [intervalo de confianza (IC) del 95\%]) y una E de 100; puntuaciones de 23 puntos o menos 
minimizan los falsos positivos $(\mathrm{E} \geq 100) \mathrm{y}$ de 25 o más puntos minimizan los falsos negativos por aparecer casi exclusivamente en sujetos sin DEM $(\mathrm{S} \geq 100)$.

En la tabla 4, observamos los valores de $S$ y E, para distintos puntos de corte del Pfeiffer; el punto de corte que maximiza $S$ y E es $10 / 6$ ( $<6$ positivo/ $\geq 6$ negativo), para el cual se obtiene una $S$ de 100 y una E de 70; puntuaciones de 5 puntos o menos minimizan los falsos positivos ( $\mathrm{E} \geq$ 100) y de 7 o más puntos minimizan los falsos negativos por aparecer casi exclusivamente en sujetos sin deterioro cognitivo $\quad(\mathrm{S} \geq 100)$; $\quad$ puntuaciones intermedias están asociadas a mayor incertidumbre diagnóstica.

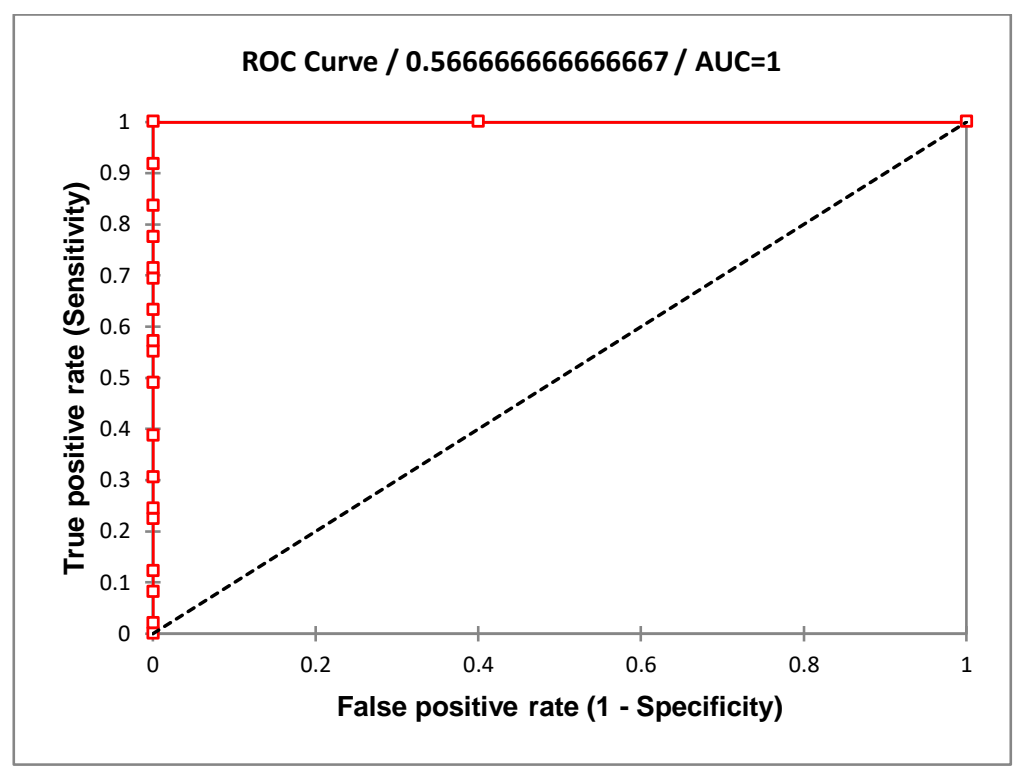

Figura 1. Curva ROC del Mini mental en Deterioro Cognitivo

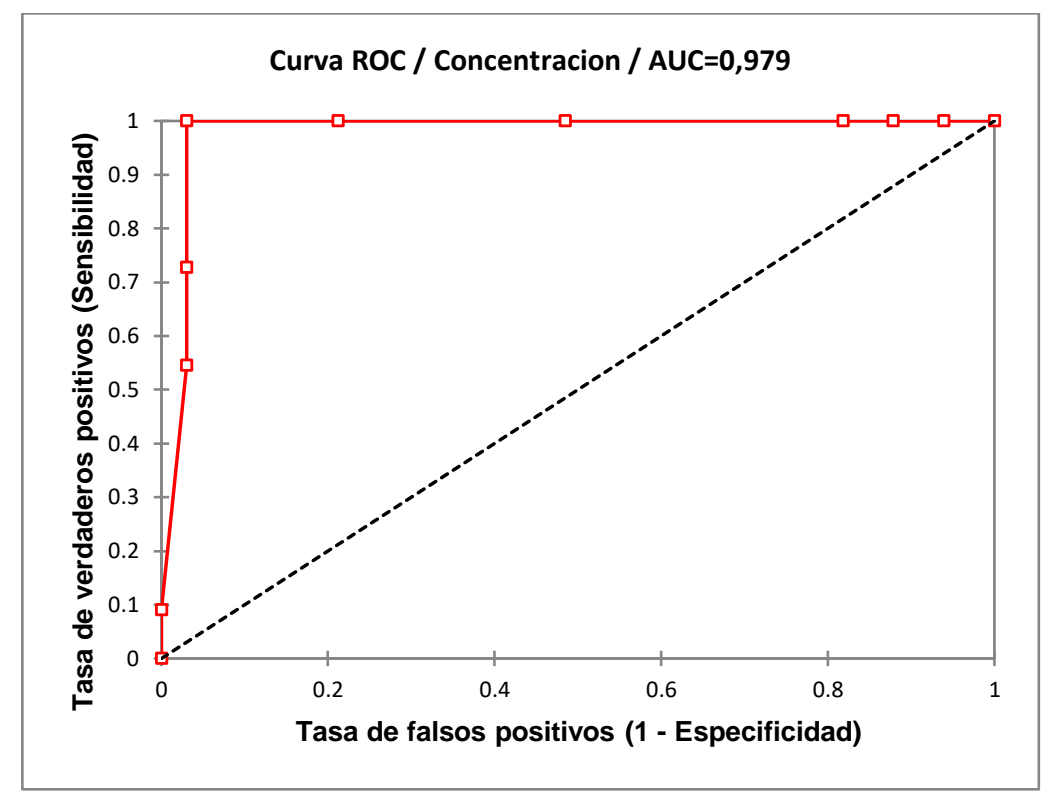

Figura 2. Curva ROC del Pfeiffer en Deterioro Cognitivo. 
En las figuras 1 y 2 se representan las curvas ROC de cada uno de los test de cribado utilizado para deterioro cognitivo, la UD del mini mental medida como área bajo la curva (AUC 1), mostrando una UD superior al test de Pfeiffer la cual muestra una (AUC 0,97).

Tabla 3. Sensibilidad y especificidad del Minimental en deterioro cognitivo.

\begin{tabular}{ccc}
\hline Mini mental & S & E \\
\hline 18 & 0,571 & 1,000 \\
19 & 0,633 & 1,000 \\
20 & 0,694 & 1,000 \\
21 & 0,714 & 1,000 \\
22 & 0,776 & 1,000 \\
23 & 0,837 & 1,000 \\
24 & $\mathbf{0 , 9 1 8}$ & $\mathbf{1 , 0 0 0}$ \\
25 & 1,000 & 1,000 \\
27 & 1,000 & 0,600 \\
28 & 1,000 & 0,000 \\
S: sensibilidad E: especificidad & & \\
\hline
\end{tabular}

Tabla 4. Sensibilidad y especificidad del Pfeiffer en deterioro cognitivo

\begin{tabular}{ccc}
\hline PFEIFFER & S & E \\
\hline 0 & 0,065 & 1,000 \\
1 & 0,129 & 1,000 \\
2 & 0,194 & 1,000 \\
3 & 0,548 & 1,000 \\
4 & 0,839 & 1,000 \\
5 & 1,000 & 1,000 \\
6 & $\mathbf{1 , 0 0 0}$ & $\mathbf{1 , 0 0 0}$ \\
7 & 1,000 & 0,565 \\
8 & 1,000 & 0,087 \\
9 & 1,000 & 0,000 \\
S: sensibilidad E: especificidad & & \\
\hline
\end{tabular}

\section{DISCUSIÓN}

$\mathrm{E}$ n la actualidad, existe una gran cantidad de métodos enfocados para la detección de DC, los test cognitivos son métodos que ha ganado terreno debido a su fácil aplicación y bajo costo, primordialmente para la atención en primer nivel; sin embargo, no se ha encontrado en la literatura médica estudios en donde se puedan comparar entre sí para demostrar su eficacia y utilidad en la práctica diaria (5). 
Se han estudiado diferentes cuestionarios, todos validados, como el AD8arg (Alzheimer Disease 8, modificado) que ayuda en el diagnóstico de DC, con características y enfoques semejantes, es importante mencionar que dentro de estos los instrumentos teóricamente aconsejables en su utilización son los que permiten la evaluación de un mayor número de dominios cognitivos (6), bajo este marco se estudiaron 55 adultos mayores del centro geriátrico de macas, en donde se observó una media de edad 82 años y el $59 \%$ perteneciente al sexo masculino.

Con respecto a las pruebas de tamizaje utilizadas, en el presente estudio se encontró que el cuestionario mini mental permitió identificar el mayor número de adultos mayores con DC con el punto de corte establecido, seguido de la prueba de Pfeiffer. Cuando se utilizó el mini mental con punto de corte de 24 o menos se reportó 50 casos, lo que equivale a $91 \%$ del universo. Para la prueba de Pfeiffer con punto de corte de 4 errores, se identificaron 32 casos positivos, lo que equivale a $58 \%$. Cabe mencionar que solo hubo una concordancia mínima entre ambos, lo que indica que son de utilidad para identificar DC, sin embargo, estos serían en poblaciones distintas.

En Ecuador se han realizado estudios sobre deterioro cognitivo en el cual podemos observar la utilización de estos cuestionarios, por ejemplo, Abambari y colaboradores en el año 2010 en una población de 391 adultos mayores del IESS; encontraron una prevalencia de deterioro cognitivo de $17,1 \%$ utilizando el cuestionario Mini mental (7).

Mientras que Guapisaca y colaboradores, en un estudio realizado en los centros geriátricos de Cuenca encontraron una prevalencia del $56 \%$ con la utilización del mismo cuestionario (8), pudiendo observar que la prevalencia de DC en pacientes institucionalizados es superior a los no institucionalizados. Además, asociado a la calidad de las relaciones familiares, el lugar donde residan las personas mayores es un factor que puede acelerar el DC (9).

Pacheco en su trabajo "repercusión del abandono en el estado mental y emocional del adulto mayor" realizado en el 2016 en el asilo de la ciudad de Cariamanga, muestra una prevalencia de DC severo de $63 \%$ y un 17\% moderado, con la aplicación del cuestionario Pfeiffer (10).

Diversos autores mencionan que a mayor edad mayor deterioro cognitivo, realidad que se evidencia también en nuestra población; a medida que se envejece la auto reparación de nuestro organismo disminuye lo que condiciona mayor riesgo de deterioro o demencia.

León y colaboradores tras evaluar 59 adultos mayores de la parroquia el salto en Babahoyo, encontraron que el DC es mayor entre los de instrucción básica 90,3 \%, y menor entre los participantes que cursaron estudios de secundaria y superior (11). Siendo estos resultados similares a los de la investigación en donde se aprecia el $65 \%$ pertenecen a instrucción primaria esto fruto de la escasa escolarización de buena parte de los ancianos de nuestro país.

En nuestro estudio se observó mayor número de individuos positivos para DC con el cuestionario mini mental en comparación con el cuestionario Pfeiffer, esto puede deberse a varios factores, la primera es el grado de escolaridad; SPMSQ es muy adecuado para pacientes con bajo nivel educativo, requiere una interpretación lingüística muy básica puede usarse en cualquier etnia y nivel socioeconómico, en comparación con el cuestionario mini mental que no puede ser aplicable a analfabetos es más extenso y complejo al exigir la utilización de lápiz y papel (8). 
Ante la ausencia de un patrón oro que permita la detección de DC, se plantea la realización de pruebas cognitivas sencillas y sensibles, como el Test de Pfeiffer o Short Portable Mental Status Questionarie (SPMSQ), usado en este estudio que permiten una primera aproximación diagnóstica de forma rápida en la atención primaria, y se confirma con una alta sensibilidad y especificidad observada. La necesidad de un entrevistador capacitado es mínima, además del tiempo mínimo de empleo por lo que podría ser incorporado como prueba rutinaria en atención primaria (12).

Carnero y colaboradores en su trabajo demuestra utilidad diagnóstica del mini mental para DC (AUC $0,84 \pm 0,02$ ) con el mejor punto de corte (22/23), llegando a la conclusión que la utilidad diagnóstica del Mini-Mental para la detección del deterioro cognitivo en Atención Primaria no es satisfactoria, a pesar del ajuste por edad y educación no mejoró la capacidad discriminativa (13).

\section{CONCLUSIONES}

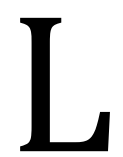

a utilidad diagnostica del cuestionario mini mental para identificar deterioro cognitivo queda una vez más entredicho AUC $1, y$ una sensibilidad y especificidad 100\%, mostrando una utilidad diagnostica superior al PFEIFFER, sin embargo, es un instrumento cuya duración y complejidad no facilita su uso como instrumento de cribado en la práctica clínica habitual (14). Además, el inconveniente de que se trata de un instrumento que evalúa DC y demencia.

- Financiación. Los autores declaran no haber recibido financiación para la realización de este artículo.
- Conflictos de interés. Los autores declaran no tener conflictos de interés.

\section{REFERENCIAS BIBLIOGRÁFICAS}

1. Medina G, Bonilla M. Relación entre deterioro cognitivo y actividad física en adultos mayores que acuden al grupo 60 y piquito de la dirección distrital 17d09 Tumbaco, Tababela, en el período octubre 2016 A noviembre 2016 [tesis doctoral]. Quito: Pontificia Universidad Católica del Ecuador. Facultad de Medicina, 2017

2. Benavidez C. Deterioro cognitivo en el adulto mayor. Revista Mexicana de Anestesiología. 2017; 40(2): 107 112

3. Gutiérrez J, Guzmán G. Definición y prevalencia del deterioro cognitivo leve. Rev Esp Geriatr Gerontol. 2017;52(1): 3-6

4. Cancino M, Rehbein L. Factores de riesgo y precursores del Deterioro Cognitivo Leve (DCL): Una mirada sinóptica. terapia psicologica 2016; 34(3):183-189

5. Contreras R, Sánchez O, Ángeles E. Comparación de los cuestionarios Carlsson-Dent y GERD-Q para detección de síntomas de enfermedad por reflujo gastroesofágico en población general. Revista de Gastroenterología de México. 2017; 82(1):19 - 25

6. Creavin ST, Wisniewski S, Noel-Storr $\mathrm{AH}$, Trevelyan CM, Hampton T, Rayment D, Thom VM, Nash KJE, Elhamoui H, Milligan R, Patel AS, Tsivos DV, Wing T, Phillips E, Kellman SM, Shackleton HL, Singleton GF, Neale BE, Watton ME, Cullum S. MiniMental State Examination (MMSE) for the detection of dementia in clinically unevaluated people aged 65 and over in community and primary care populations. Cochrane Database of Systematic Reviews 2016

7. Abambari C, Atiencia R, Arevalo C. Riesgo de demencia y factores asociados en adultos mayores IESS. 
Cuenca, 2009. Universidad de Cuenca. Facultad de Ciencias Médicas. Escuela de Medicina. 2010. Disponible en: http://dspace.ucuenca.edu.ec/bitstrea m/123456789/3505/1/doi335.pdf

8. Guapisaca D, Guerrero J, Idrovo P. Prevalencia y factores asociados al deterioro cognitivo en adultos mayores de los asilos de la ciudad de Cuenca. 2014. Universidad de cuenca. Facultad de ciencias médicas. Escuela de medicina. 2014. Disponible en: http://dspace.ucuenca.edu.ec/bitstrea m/123456789/20233/1/TESIS.pdf?fb clid=IwAR39DjUa18jwMCUZ6UeFmC C_crKtOXFidSSB0jvk6ACPauDKqcMXe xmSX70

9. Leiva A, Sánchez JL, León J, Palacios L. Factores predictores de deterioro cognitivo en población mayor de 64 años institucionalizada y no institucionalizada. Enferm Clin. 2016;26(2):129-136

10. Pacheco M. Repercusión del abandono en el estado mental y emocional del adulto mayor que reside en el asilo de ancianos hogar san José de la ciudad de Cariamanga. Universidad Nacional de Loja. Área de la salud humana.
Carrera de medicina humana. 2016. Disponible en: http://dspace.unl.edu.ec/jspui/bitstre am/123456789/9878/1/TESIS\%20M ANUEL\%20ANIBAL\%20PACHECO\%2 OSANCHEZ.pdf

11. León G. Características del deterioro cognitivo en el adulto mayor de la parroquia El Salto. Babahoyo Ecuador. Centro de biotecnología. 2016; 5(1): 31 - 39

12. Vega $T$, Miralles $M$, Mangas $J$, Castrillejo D, Rivas A, Gil M, López A, Arrieta E, Lozano J, Fragua M. Prevalencia de deterioro cognitivo en España. Estudio Gómez de Caso en redes centinelas sanitarias. Neurología. 2018; 33(8): 491-498

13. Carnero C, Cruz I, Espejo B, Cárdenas S. Efectividad del Mini-Mental en la detección del deterioro cognitivo en Atención Primaria. Aten Primaria. 2013;45(8):426---433

14. Carnero C, Sáez C, Montiel I, del Saz P, Feria I, Pérez M, Ruiz J, Vílchez R, Montoro T. Utilidad diagnóstica del Test de las Fotos (Fototest) en deterioro cognitivo y demencia. Neurología 2007;22(10):860-869 